\title{
A CLASS OF UNIFORM CONVERGENCE STRUCTURES
}

\author{
G. D. RICHARDSON
}

Abstract. In 1967, Cook and Fischer introduced in the journal Mathematische Annalen the notion of a uniform convergence structure, abbreviated u.c.s., for a set $X$. Here we consider the class $\Gamma$ of u.c.s. which have the following property: a u.c.s. $I \in \Gamma$ provided there is a filter $\Phi \in I$ such that $\mathcal{F}$ is finer than $\Phi(x)$ for every filter $\mathcal{F}$ which converges to $x$, for each $x \in X$. Various properties of the class $\Gamma$ are discussed. The main result is that a topology $\tau$ for $X$ is regular if and only if there is an $I \in \Gamma$ such that $I$ induces $\tau$. Also it it is shown that each $I \in \Gamma$ induces a regular topology for $X$.

The class $\Gamma_{0}$ of u.c.s. which satisfy the completion axiom was first introduced by Biesterfeldt, Indag. Math., 1966. Here it is shown that $\Gamma_{0} \subset \Gamma$ and a characterization of the class $\Gamma_{0}$ is given in terms of Cauchy filters.

1. Introduction. The reader is referred to [3] and [4] for definitions and notation used here.

Let $X$ be any set. Denote by $\Gamma$, the class of all uniform convergence structures, abbreviated u.c.s., for $X$ with the following property: for each $I \in \Gamma$ there is a $\Phi \in I$ such that $\tau_{I}(x)$ is the collection of all filters on $X$ which are finer than $\Phi(x)$, for each $x \in X$.

Various properties of the class $\Gamma$ are discussed. The main result is that any topology for $X$ is regular if and only if there is a u.c.s. in $\Gamma$ that induces the given topology.

Finally the u.c.s. for $X$ which satisfy the completion axiom are discussed. The completion axiom was first introduced in [1].

2. A characterization for regular topologies. Let $I \in \Gamma$. Then there is a symmetric filter $\Phi \in I$ with $\Phi \leqq[\Delta]$ ( $\Phi$ coarser than the diagonal filter) such that $\tau_{I}(x)$ is the collection of all filters on $X$ which are finer than $\Phi(x)$.

\section{Proposition 1. Let $I \in \Gamma$. Then $\tau_{I}$ is a topology for $X$.}

Proof. From [2], we must show that for $A(x) \in \Phi(x), A=A^{-1} \in \Phi$, there is an $H \in \Phi(x)$ such that for each $y \in H, A(x) \in \Phi(y)$. Since $\Phi^{2}(x)$ converges to $x$, we have that $\Phi^{2}(x)=\Phi(x)$. Hence there is a $B \in \Phi$ such that $B^{2}(x) \subset A(x)$. Let $H=B(x) \in \Phi(x)$ and let $y \in H$. We claim that $A(x) \supset B(y) \in \Phi(y)$. If $z \in B(y)$, then $(y, z) \in B$. Since $y \in B(x)$, then $(x, y) \in B$ and it follows that $(x, z) \in B^{2}$ or $z \in B^{2}(x) \subset A(x)$.

Received by the editors September 9, 1969.

AMS Subject Classifications. Primary 5422, 5410; Secondary 5420.

Key Words and Phrases. Uniform convergence structures, symmetric filters, diagonal filters, ultrafilters, Cauchy filters, regular topologies. 
Hence the claim follows and thus $\tau_{I}$ is a topology for $X$.

The proof of the following proposition is similar to that in [2] for uniform spaces and will be deleted here.

Proposition 2. Let $I \in \Gamma$ and $A \subset X, B \subset X \times X$. Then $\mathrm{Cl}(A)=$ $\bigcap_{V \in \Phi} V(A)$ and $\mathrm{Cl}(B)=\bigcap_{v \in \Phi} V \circ B \circ V$.

Corollary. If $I \in \Gamma$, then $\mathrm{Cl}(\Phi) \in I$.

Proof. From Proposition 2, $\mathrm{Cl}(\Phi) \geqq \Phi^{3}$ and hence $\mathrm{Cl}(\Phi) \in I$.

Proposition 3. If $I \in \Gamma$, then $\tau_{I}$ is a regular topology.

Proof. We claim that $x \times \mathrm{Cl}(\Phi(x)) \geqq \mathrm{Cl}(\Phi) \in I$ ( $\dot{x}$ is a fixed ultrafilter); of course, it suffices to show that $\{x\} \times \mathrm{Cl}(A(x)) \subset \mathrm{Cl}(A)$ where $A \in \Phi$. If $y \in \mathrm{Cl}(A(x))$ and $B \in \Phi$, then $(B(x) \times B(y)) \cap A \neq \varnothing$. Hence $(x, y) \in \mathrm{Cl}(A)$ and the claim follows. Therefore $\tau_{I}$ is a regular topology.

TheOREm. Let $(X, \tau)$ be a topological space with $\eta(x)$ denoting the neighborhood filter at $x \in X$. Then $\tau$ is regular iff there is an $I \in \Gamma$ inducing $\tau$.

Proof. Let $I \in \Gamma$ such that $\tau_{I}=\tau$. Then by Proposition 3, $\tau$ is regular.

Conversely, assume that $\tau$ is a regular topology. Denote by $\Psi_{0}=\bigvee_{z \in X}(\dot{z} \times \eta(z)), \Phi=\Psi_{0} \wedge \Psi_{0}^{-1}, F(X \times X)$ the collection of all filters on $X \times X$, and $B=\left\{\Phi^{n} \mid n=1,2, \cdots\right\}$. Clearly $B$ is a base for a u.c.s. $I$ for $X$. That is, $I=\left\{\Psi \in F(X \times X) \mid \Psi \geqq \Phi^{n}\right.$ for some $\left.n=1,2, \cdots\right\}$ is a u.c.s. for $X$.

We claim that $\tau_{I}=\tau$. The regularity of $\tau$ implies property (3) of Theorem 1 of [5]. Hence from property (2) of the same theorem, we have that $\Phi(x)=\eta(x)$ for each $x \in X$. Thus $\dot{x} \times \eta(x)=\dot{x} \times \Phi(x) \geqq \Phi$ and hence $\tau(x) \subset \tau_{I}(x)$.

Conversely, if $\mathscr{F} \in \tau_{I}(x)$, then $\dot{x} \times \mathfrak{F} \geqq \Phi^{n}$ for some positive integer $n$. Hence $\mathcal{F}=(\dot{x} \times \mathfrak{F})(x) \geqq \Phi^{n}(x)$. Thus we must show that $\Phi^{n}(x) \geqq \eta(x)$. Assume $n \geqq 2$. Let $N \in \eta(x)$ be open. Using the regularity of $\tau$, there exists open neighborhoods $N_{i} \in \eta(x)(i=1,2, \cdots, n)$ such that $x \in N_{1} \subset \mathrm{Cl}\left(N_{1}\right) \subset N_{2} \subset \mathrm{Cl}\left(N_{2}\right) \subset \cdots \subset \mathrm{CL}\left(N^{n}\right) \subset N$. For each $z \in X$, define

$$
\begin{aligned}
N_{z} & =N_{1} \quad \text { for } z \in N_{1}, \\
& =N_{2} \quad \text { for } z \in \mathrm{Cl}\left(N_{1}\right)-N_{1}, \\
& =N_{k+1}-\mathrm{Cl}\left(N_{k-1}\right) \quad \text { for } z \in \mathrm{Cl}\left(N_{k}\right)-N_{k} \quad(k=2,3, \cdots, n-1), \\
& =N_{k+1}-\mathrm{Cl}\left(N_{k}\right) \quad \text { for } z \in N_{k+1}-\mathrm{Cl}\left(N_{k}\right) \quad(k=1,2, \cdots, n-1), \\
& =N-\mathrm{Cl}\left(N_{n-1}\right) \quad \text { for } z \in \mathrm{Cl}\left(N_{n}\right)-N_{n}, \\
& =X-\mathrm{Cl}\left(N_{n}\right) \quad \text { for } z \in X-\mathrm{Cl}\left(N_{n}\right) .
\end{aligned}
$$


We claim that

$$
\left[\left(\bigcup_{z \in X}\left(\{z\} \times N_{z}\right)\right) \cup\left(\bigcup_{z \in X}\left(\{z\} \times N_{z}\right)\right)^{-1}\right]^{n}(x) \subset N .
$$

Let $y \in$ L.H.S., $z_{0}=x, z_{n}=y$, and $A$ equal the set in brackets. Hence $\left(z_{i-1}, z_{i}\right) \in A$ for some $z_{i} \in X(i=1,2, \cdots, n)$. By computation, one can show that $z_{i} \in N_{i+1}(i=1,2, \cdots, n-1)$ and $y \in \mathrm{Cl}\left(N_{n}\right) \subset N$. Therefore our claim follows and we have that $\Phi^{n}(x)=\eta(x)$ for each natural number $n$ and each $x \in X$. Thus $\tau_{I}=\tau$.

Proposition 4. If $\tau$ is a compact Hausdorff topology for $X$, then there is exactly one $I \in \Gamma$ inducing $\tau$.

Proof. From [2] we have that $I=\{\Phi \in F(X \times X) \mid \Phi \geqq \mathcal{U}\}$, where $\mathcal{U}=\{$ all neighborhoods of $\Delta\}$, induces $\tau$. Of course $I \in \Gamma$. Hence if $I_{1} \in \Gamma$ and induces $\tau$, then we want to show that $I=I_{1}$.

Let $\Phi_{1} \in I_{1}$ be a symmetric filter such that $\tau(x)$ is the collection of all filters on $X$ which are finer than $\Phi_{1}(x)$. We claim that $u \geqq \Phi_{1} \circ \Phi_{1}$. Let $A_{1}=A_{1}^{-1} \in \Phi_{1}$. Since $A_{1}(x) \times A_{1}(x) \subset A_{1} \circ A_{1}$ for each $x \in X$, we have that $\mathrm{U}_{x \in X}\left(A_{1}(x) \times A_{1}(x)\right) \in \mathcal{U}$ and is contained in $A_{1} \circ A_{1}$. Hence the claim follows and thus $I \subset I_{1}$.

Conversely, we claim that $\Phi_{1} \geqq \mathcal{U}$. Suppose there is a $V \in \mathcal{u}$ such that for all $A_{1} \in \Phi_{1}, A_{1} \cap V^{c} \neq \varnothing$. Assume w.l.o.g. that $V$ is an open neighborhood of $\Delta$. The set $\left\{A_{1} \cap V^{c} \mid A_{1} \in \Phi_{1}\right\}$ is a base for a filter $\mathscr{L}$ on $X \times X$. Since $(X \times X, \tau \times \tau)$ is compact, $(x, y) \in$ adh $(\mathscr{L})$ for some $x, y \in X$. Hence $(x, y) \in \mathrm{Cl}\left(A_{1} \cap V^{c}\right)$ for each $A_{1} \in \Phi_{1}$. Thus $(x, y)$ $\in \mathrm{Cl}\left(V^{c}\right)=V^{c}$ and $(x, y) \in \mathrm{Cl}\left(A_{1}\right)$ for each $A_{1} \in \Phi_{1}$. Since $\Delta C V, x \neq y$. Also $\mathrm{Cl}\left(\Phi_{1}\right)(x)=\Phi_{1}(x)$ and we have that $x \in \mathrm{Cl}(\{y\})$. This contradicts $\tau$ being Hausdorff. Hence $\Phi_{1} \geqq \mathcal{U}$ and thus $\Phi_{1} \in I$. Let $\Psi \in I_{1}$. Then of course $\Psi \geqq \Psi \wedge \Psi^{-1} \wedge \Phi_{1} \in I_{1}$. By an identical argument just given for $\Phi_{1}$, we have that $\Psi \wedge \Psi^{-1} \wedge \Phi_{1} \geqq \mathcal{U}$. Hence $\Psi \geqq \mathcal{U}$ and thus $I=I_{1}$.

3. Completion axiom. The following definition is easily seen to be equivalent to that given in [1]. A u.c.s. $I$ is said to satisfy the completion axiom, abbreviated c.a., provided there is a base for $I$ consisting of symmetric filters coarser than the diagonal filter such that for each Cauchy filter $\mathcal{F}$ on $X, \mathcal{F} \times \mathfrak{F} \geqq \Phi$ for every $\Phi$ in the base.

Let $I$ satisfy the c.a. with base $B$.

Proposition 5. If I satisfies the c.a. and $\Phi \in B$, then $\tau_{I}(x)$ is the collection of all filters on $X$ which are finer than $\Phi(x)$.

Proof. Clearly $\Phi(x) \in \tau_{I}(x)$. If $\mathscr{F} \in \tau_{I}(x)$, then $\mathcal{F} \wedge \dot{x} \in \tau_{I}(x)$. Let $A \in \Phi$. Since $I$ satisfies the c.a., then $(F \cup\{x\}) \times(F \cup\{x\}) \subset A$ for 
some $F \in \mathcal{F}$. Hence $F \subset A(x)$, which implies that $\mathcal{F} \geqq \Phi(x)$ and thus the proposition follows.

Let $\Gamma_{0}$ denote the collection of u.c.s. for $X$ which satisfy the c.a. From the above proposition $\Gamma_{0} \subset \Gamma$. Hence each $I \in \Gamma_{0}$ induces on $X$ a regular topology.

Let $C_{I}$ denote the collection of all Cauchy filters on $X[3]$.

Proposition 6. If I is any u.c.s. for $X$, then $I \in \Gamma_{0}$ iff $\bigwedge_{\mathfrak{F} \in \mathfrak{e}_{I}}(\mathfrak{F} \times \mathcal{F}) \in I$.

Proof. Clearly the necessity follows. Conversely, if $\Lambda_{\mathfrak{F} \in \mathfrak{e}_{I}}(\mathfrak{F} \times \mathfrak{F})$ $\in I$ then let $B=\left\{\Phi \in I \mid \Phi=\Phi^{-1}, \Phi \leqq \Lambda_{\mathfrak{F} \in \mathfrak{e}_{I}}(\mathfrak{F} \times \mathfrak{F})\right\}$. Since $\dot{x} \in \mathbb{C}_{I}$ for each $x \in X$, then $\Phi \leqq[\Delta]$ for each $\Phi \in B$. Clearly $B$ is a c.a. base for $I$.

I conjecture that each $I \in \Gamma_{0}$ induces a completely regular topology on $X$.

\section{REFERENCES}

1. H. J. Biesterfeldt, Completion of a class of uniform convergence spaces, Nederl. Akad. Wetensch. Proc. Ser. A 69 = Indag. Math. 28 (1966), 602-604. MR 34 \#5052.

2. N. Bourbaki, General topology. Part I, Hermann, Paris and Addison-Wesley, Reading, Mass., 1966. MR 34 \#5044a.

3. C. H. Cook and H. R. Fischer, Uniform convergence structures, Math. Ann. 173 (1967), 290-306. MR 36 \#845.

4. H. R. Fischer, Limesräume, Math. Ann. 137 (1959), 269-303. MR 22 \#225.

5. D. C. Kent, A note on pretopologies, Fund. Math. 62 (1968), 95-100. MR 36 \#7102.

East Carolina University, Greenville, North Carolina 27834 